\title{
Internacionalización en el sector aéreo de América Latina: estudio multicaso de Aeroméxico, Avianca y Latam (Internationalization in the Latin American air sector: multi- case study of Aeromexico, Avianca and Latam)
}

\author{
José Satsumi Lopez Morales • \\ Mauricio Alejandro Montañez Cuevas * \\ Jorge Antonio Zertuche Zertuche * \\ Daniel Gregorio Paez Aguirre * \\ Alejandro Martinez Cespedes ^
}

\begin{abstract}
The objective of this paper is to analyze the internationalization strategies of the Latin American airlines: Aeromexico (Mexico), Avianca (Colombia) and LATAM (Chile). For this, a qualitative multi-case study technique was applied. The research question that is intended to answer in this paper is: in what way are the main airlines in Latin America internationalized? The results suggest that the airlines studied use similar internationalization strategies, which are based on strategic alliances with other airlines in order to offer a better service to their passengers. They were also identified as the main characteristics of these airlines that are strong in their domestic market (especially Aeromexico) and then they start to expand their operations abroad. Likewise, Avianca and LATAM have the main market in Latin America, while Aeromexico has a greater presence in the United States.
\end{abstract}

Key words: Aeromexico, airline industry, Avianca, internationalization, LATAM, service sector

JEL: F23, M16.

\footnotetext{
- Tecnológico Nacional de México - Instituto Tecnológico de Veracruz / División de Negocios, Universidad de Monterrey. Email: jsatsumi@gmail.com

* División de Negocios Universidad de Monterrey. Email: mauricio.montanez@udem.edu

* División de Negocios Universidad de Monterrey. Email: jorge.zeruche@udem.edu

* División de Negocios Universidad de Monterrey. Email: daniel.paez@udem.edu

- División de Negocios Universidad de Monterrey. Email: alejandro.martinezc@udem.edu
} 
Resumen. El objetivo de este trabajo es analizar las estrategias de internacionalización de las aerolíneas latinoamericanas Aeroméxico (México), Avianca (Colombia) y LATAM (Chile). Para esto se aplicó una técnica cualitativa de estudio multi-caso. La pregunta de investigación que se pretende responder en este trabajo es ¿de qué manera se internacionalizan las principales aerolíneas en América Latina?. Los resultados sugieren que las aerolíneas estudiadas utilizan estrategias similares de internacionalización, las cuáles están basadas en alianzas estratégicas con otras aerolíneas para poder ofrecer un mejor servicio a sus pasajeros. Además fueron identificadas como principales características de estas aerolíneas que son fuertes en su mercado interno (sobre todo Aeroméxico) y de ahí parten para poder expandir sus operaciones hacía el exterior. Asimismo Avianca y LATAM tiene como principal mercado América Latina, en cambio Aeroméxico tiene una mayor presencia en Estados Unidos.

Palabras clave: Aeroméxico, Avianca, internacionalización, LATAM, sector aéreo, sector servicios

\section{Introducción}

El objetivo de este trabajo es analizar la estrategias de internacionalización de las tres principales aerolíneas latinoamericanas: Aeroméxico (México), LATAM (Chile) y Avianca (Colombia), para determinar cuáles fueron usadas para lograr el crecimiento y desarrollo que han tenido en los últimos años. El sector servicios es cada vez más importante en el desarrollo de la economía de los países, así como en las empresas, ya que para ser más competitivos este sector genera empleos y ayuda a mejorar la calidad de vida de la sociedad (Ministerio de Comercio, 2011).

Históricamente el estudio del sector servicios ha sido limitado (Gleich, Schmeisser \& Zschoche, 2016) sin embargo en los últimos años este se ha incrementado. Pese a este incremento la comprensión del proceso de internacionalización en este sector está en las primeras etapas, sobre todo se encuentran menos desarrollada en las empresas de servicios originarias de regiones emergentes como América Latina (Lopez- Morales, 2018) , ya que se identificaron estudios previos enfocados en el sector servicios, pero utilizando como muestra empresas multinacionales de países desarrollados, principalmente de Estados Unidos, Europa y Japón (Driffield, Pereira \& Temouri, 2017; Wang \& Chen, 2015; Li \& Guisinger, 1992). 
Asimismo cada día existen más opciones de servicios, sobretodo en el sector aéreo, específicamente las aerolíneas cumplen con una doble función de permitir a las empresas vender sus bienes y facilitar los flujos turísticos (CEPAL, 2017). Por eso hoy en día las aerolíneas han implementado diferentes estrategias para aumentar su mercado, ofrecer mayores frecuencias de vuelos y un mejor servicio a los pasajeros. En este contexto para este estudio se consideraron las aerolíneas latinoamericanas: Aeroméxico (México), Avianca (Colombia) y LATAM (Chile), ya que estas son las que tienen mayores aerolíneas en movimiento de pasajeros de la región (Banco Interamericano de Desarrollo, 2016).

Además en el sector aéreo el factor tecnológico es muy importante ya que los aviones son más modernos y más rápidos. Además el servicio al cliente ha mejorado al ofrecer mejor calidad de servicio por medio del internet (OMC, 2005). La mayoría de las aerolíneas se pueden considerar como empresas que nacieron globales debido a las características de este mercado, forzándolas a tener, desde sus inicios, operaciones internacionales (Cruz-García \& Puerta, 2012).

Este trabajo se estructura como sigue: primero se presenta la revisión de literatura, segundo se presenta el método utilizado, segundo se presenta la situación general de Aeroméxico, LATAM y Avianca, donde se hablará acerca de los puntos más importantes, así como de su flota de aviones y las frecuencias de vuelos internacionales. Posteriormente se presenta el análisis de resultados, en donde se analizan las diferentes estrategias utilizadas por las aerolíneas estudiadas. A continuación se discuten los resultados obtenidos del trabajo y finalmente se presentan las conclusiones derivadas del análisis de resultados.

\section{Revisión de literatura}

\section{Teorías de internacionalización}

Una de los principales autores que estudian la internacionalización ha sido John H. Dunning. Él ha contribuido con la Teoría OLI. El nombre OLI viene de los 3 factores más importantes que determinan las actividades internacionales de las compañías multinacionales: propiedad (0), localización (L) e internalización (I) (Eden \& Dai, 2010). 
La teoría de Dunning surgió de la pregunta ¿por qué las empresas invierten en el exterior, o generalmente, cuál es la cantidad y composición de la producción internacional?. El paradigma ecléctico se basa en el supuesto de que las empresas tiene el propósito principal de determinar si una decisión de internacionalización ofrece a una empresa más valor que otras opciones disponibles.

Por su parte, Rugman (2010) explica esta teoría al enseñar que en orden de poder desarrollar y crecer en el mercado globalizado las compañías tienen que tener una ventaja de propiedad, ubicación ventajosa y una ventaja de internalización. Esta teoría provee un marco para todas las compañías que internacionalizan. Las compañías deben asegurar que esas ventajas se han llevado a cabo para determinar si es beneficio para ellos realizar una inversión extranjera directa (Dunning, 1998).

La segunda teoría es mejor conocida como el "Modelo de Uppsala", desarrollada en la Universidad de Uppsala en Suecia. Esta fue publicada por primera vez en 1977 (Johanson \& Valhne, 1977). Como describieron Gustafsson y Zasada en el 2011, este modelo fue considerado como uno de los modelos de la etapa primaria de internacionalización en el mundo moderno. La idea principal de este modelo es que la compañía aumentará gradualmente sus recursos planeados a un país en particular conforme adquiere experiencia de las actividades realizadas en ese mercado (LópezMorales \& Nava- Aguirre, 2018).

Este es un modelo de internacionalización el cual se basa en el aprendizaje y en adquirir conocimiento (Danciu, 2012). Esta teoría, asume que el conocimiento acerca de la internacionalización y del mercado internacional es importante para el éxito en el extranjero. Cuanto más conocimiento y experiencia adquiere la empresa más obstáculos van a superarse, lo que significa que las empresas tienen que desarrollarse en su organización interna, así como actualizar sus procesos de negocios.

Ya este modelo se ha ido actualizando durante los últimos años, por lo que sus autores Johanson y Vahlne (1977) proponen cuatro etapas en las cuales explican como las oportunidades de aprendizaje afectan el entorno de la empresa. Las cuatro etapas consisten en: (1) el conocimiento y las oportunidades que la compañía puedan detectar en el entorno internacional, (2) las decisiones de compromiso en la relación (3) el aprendizaje, creación y 
construcción de pruebas, y finalmente (4) el logro de la adquisición de una posición de redes (Alserus \& Tykesson, 2011).

\section{Internacionalización de servicios}

La internacionalización de los servicios es un fenómeno reciente en comparación con la manufactura (Rammal \& Rose, 2014). Asimismo los avances tecnológicos de los últimos años han sido un detonador importante en el proceso de expansión hacía mercados internacionales de empresas del sector servicios.

El Acuerdo General sobre Comercio de Servicios que está inserto en la Organización Mundial de Comercio (OMC) identifica cuatro formas en las que los servicios pueden internacionalizarse. Estas formas son: 1) Comercio transfronterizo, 2) Consumo en el exterior, 3) Presencia comercial ,4) Presencia de personas físicas (World Trade Organization, 2013). Estas formas de internacionalización brindan un importante marco para analizar la expansión internacional de empresas del sector servicios.

Asimismo, en el caso de los servicios la calidad es un factor muy importante por ello es importante controlar adecuadamente la prestación de los servicios. Este control es probable que influya en la selección de la inversión extranjera directa como forma de internacionalización (FrancoArroyave, Martins- Cheze, Siegel, Díaz- Vázquez, 2014).

Si bien se ha incrementado la literatura sobre la internacionalización de servicios, sigue siendo predominante los estudios en el sector manufacturero. Capar y Kotabe (2003) analizan la relación multinacionalidad y desempeño en empresas de servicios. Además Rivas y Mayorga (2011) analizan las formas de internacionalización en 16 restaurantes de origen peruano. Faulconbridge y Muzio (2016) estudian la internacionalización de empresas inglesas que brindan servicios jurídicos hacia Italia.

\section{Método}

El trabajo que se presenta a continuación es un estudio multi-caso, ya que el método del caso es una herramienta importante de investigación. Su principal fortaleza radica en que profundiza en el comportamiento de las personas y los hechos (Yin, 2009). La pregunta de investigación que se pretende 
responder en este trabajo es ¿de qué manera se internacionalizan las principales aerolíneas en América Latina?.

En este trabajo fue utilizada la metodología para la elaboración de casos de estudio propuesta por Reddy \& Agarwal (2012). De acuerdo a esta metodología este trabajo sería un caso tipo II, ya que estará basado en fuentes secundarias y no primarias. Las principales fuentes de información secundarias son: periódicos, revistas, entrevistas en los medios, páginas de internet y noticias de la industria.

En primera instancia se seleccionó un tema de estudio específico de las empresas objeto de estudio, en este caso la internacionalización. Asimismo las principales fuentes de información fueron: la página web institucional, otros casos de estudio, páginas web de periódicos nacionales e internacionales, páginas web de otras empresas del sector y/o relacionadas con el sector, así como entrevistas. La información de las fuentes mencionadas que se consideró para este estudio es aquella que sí representaba una forma de internacionalización, tal como: las alianzas estratégicas, operaciones internacionales, fusiones, códigos compartidos y frecuencias de vuelo semanales a destinos internacionales.

\section{Situación general de las compañías}

\section{Aeromexico}

En 1934, Antonio Díaz Lombardo fundó Aeronaves de México en donde el vuelo inaugural fue de Ciudad de México a Acapulco con un avión Stinson piloteado por Julio Zinser. Debido a la escasez de partes y refacciones de aviones tras la 2da guerra mundial en 1941, Aeronaves de México tuvo que vender el $25 \%$ a Pan American World Airways.

En diciembre de 1959 se adoptó al Caballero Águila como emblema de la compañía y los aviones fueron llamados con el nombre de guerreros aztecas. En 1960 se firmó un contrato con la fábrica Douglas para adquirir aviones DC-8, ya que para volar a Canadá se le exigió a Aeronaves de México aviones de largo alcance. La empresa cambia el nombre a Aeroméxico en 1971 con el nuevo Director General Raymundo Cano Pereira. El 1 de octubre de 1988 nace la empresa Aerovias de México SA de CV, comenzando la operación con 25 aviones y con 3,500 empleados. 
Asimismo en el año 2000 nace Skyteam, una alianza de códigos compartidos entre aerolíneas donde los miembros fundadores son Aeroméxico, Air France, Delta y Korean Air ampliando sus redes de destinos y agregando beneficios a los clientes.

En abril del 2011 Aeroméxico comenzó a cotizar en la Bolsa Mexicana de Valores tras fijar un precio de salida para sus títulos de $\$ 31$ pesos ( $\$ 2.62$ USD).En el 2013 Aeroméxico suma siete nuevos Boeing 787-8 Dreamliner, un modelo de avión de los más modernos e innovadores del mercado (https://aeromexico.com).

Actualmente Aeroméxico ofrece vuelos a 45 países distintos en tres continentes (García, 2017). Lo anterior has sido gracias a la nueva flota que cuenta con más de 130 aviones, incluyendo los 69 Boeing que son fabricados en Estados Unidos y los 63 Embraer hechos en Brasil (https://world.aeromexico.com).

\section{LATAM}

El primer antecedente de LATAM fue LAN, fundada en 1929 por el Comandante Arturo Merino Benito en Chile. En 1961 fue fundada TAM (Taxi Aéreo Marília) por cinco pilotos de vuelos chárter (https://www.latam.com). La primera ruta de LAN dentro de Sudamérica fue a Buenos Aires, Argentina en 1946, y en Miami hacia los Estados Unidos en 1958. La primera ruta fuera del continente fue Europa con destinos a las ciudades de Madrid, París y Frankfurt en 1970 (Delpiano, 2009).

En 1997 LAN lista sus acciones en la bolsa de Nueva York, siendo la primera aerolínea latinoamericana (LATAM, 2014). En 1999 empezó las operaciones de LAN Perú (LAN, 2013). En el año 2000 LAN se incorpora a la alianza OneWorld. En 2003 inicio operaciones LAN Ecuador.

En 2005 empezaron las operaciones de LAN Argentina. En 2006 LAN empieza a cotizar en la bolsa de Nueva York. En el 2010 LAN forma oficialmente parte de la alianza Star Alliance (https://www.latam.com), en 2011 empezó las operaciones de LAN Colombia (LAN, 2013).

En el año 2012 nace el Grupo de LATAM Airlines, como resultado de la fusión entre las aerolíneas LAN Airlines y TAM Linhas aéreas de Brasil (LATAM, 2016). Esta fusión posiciono a la aerolínea como la más importante de América Latina. Asimismo se formó con el objetivo de tener más 
disponibilidad de vuelos y ser una de las aerolíneas más importantes en el mundo (Ruiz, 2012).

En la actualidad la aerolínea LATAM suma alrededor de 40 mil empleados (https://www.latam.com) alrededor de 338 aviones, ofreciendo servicios de transportes para pasajeros alrededor de 135 destinos en 22 países y servicios de carga a alrededor de 134 destinos en 23 países (LATAM, 2015).

\section{AVIANCA}

Avianca fue fundada en Colombia el 5 de diciembre de 1919 y en 1921 comenzaron con las rutas entre Barranquilla, Girardot y Neiva. En 1946, Avianca abrió nuevas rutas, comenzando a expandirse internacionalmente, las rutas que abrieron fue a Quito, Lima, Panamá, Miami, Nueva York y algunas ciudades de Europa. En 1981, lograron la ventaja competitiva de tener la Terminal Puente Aéreo, en la cual tenían la oportunidad de mejorar la experiencia del cliente en el aeropuerto así como en el avión, esta Terminal operaba las rutas de Miami, Nueva York, Medellín, Cali, Pasto y Montería (http://www.aviancacargo.com).

En 1994 Avianca logró una alianza estratégica muy importante con dos aerolíneas muy importantes en Colombia. Esta alianza estaba conformada por Avianca, SAM (Sociedad Aeronáutica de Medellín) y HELICOL (Helicópteros Nacionales de Colombia), esta alianza fue llamada Sistema Avianca. En el año del 2002 Avianca y SAM (Sociedad Aeronáutica de Medellín) realizaron una nueva alianza con Aces (Aerolíneas Centrales de Colombia) Ilamándola Alianzas Summa, después decidieron liquidar la alianza para invertir y fortalecer a Avianca.

En el año del 2009 Avianca realiza una fusión con la aerolínea centroamericana TACA, formando Avianca - Taca, esta fusión se realiza con el objetivo de tener una mejor posición en el mercado y así tener una mejor capacidad de crecimiento en el sector (http://www.aviancacargo.com).

En el año del 2011 lanzaron el programa para viajeros "LifeMiles" y hoy en día tiene más de 7 millones de viajeros en el programa, este programa consiste en acumular millas durante los vuelos y así obtener beneficios en los viajes, en las compras, con las tarjetas de crédito o débito y el programa de coinversión. Ese mismo año Avianca inauguró 12 nuevas 
rutas y se incrementaron 155 frecuencias de vuelos teniendo más de 100 destinos en América y Europa logrando 4000 vuelos por semana. Asimismo la aerolínea busco fortalecer el sector de carga ampliando las bodegas y comprando aviones con capacidad de 70 toneladas.

En el año 2012 Avianca ingreso a la alianza global "Star Alliance". Esta alianza ofrece grandes beneficios para los pasajeros como salas VIP en los aeropuertos más importantes, conexiones alrededor del mundo, servicios de prioridad en el check- in, reservas entre otros. Esta alianza ha ganado diferentes premios y reconocimientos como el premio de liderazgo mundial de transporte aéreo de mercado y la mejor alianza aérea (http://www.avianca.com).

En el año del 2013 Avianca cambió su nombre a Avianca Holding S.A. y comenzó a cotizar en la Bolsa de Nueva York. En la actualidad Avianca cuenta con más de 6000 vuelos semanales, 172 aviones, 106 destinos en 26 países y más de 7 millones de viajeros en su programa LifeMiles (http://aviancaholdings.com).

\section{Análisis de resultados}

\section{Aeroméxico}

Aeroméxico creó la alianza estratégica con Air France, Delta y Korean Air llamada Skyteam en el 2000 con el propósito de aumentar su ventaja competitiva sobre otras aerolíneas y generar beneficios mutuos entre estas gracias a los códigos compartidos. Con el paso del tiempo, se fueron sumando más aerolíneas, en la mayoría de los casos la aerolínea con mayor prestigio de cada país (https://www.skyteam.com/es/about/).

Actualmente son 20 aerolíneas afiliadas a Skyteam de las cuales nueve comparten los códigos compartidos. Se cuenta con 1,074 destinos, 177 países y 16,609 salidas diarias. También con esta alianza los clientes de Aeroméxico pueden tener fácil acceso a distintos destinos internacionales. Las aerolíneas que forman parte de Skyteam son Aeroflot, Aerolineas Argentinas, AirFrance, Alitalia, China Airlines, China Eastern, China Southern, CzechAirlines, Delta Airlines, Garuda Indonesia, Kenya Airways, KLM, Korean Air, Korean Air, Middle East Airline, Saudia, Tarom, Vietnam Airlines y Xiamen Air. Además Aeroméxico cuenta con alianzas con otras 
aerolíneas que no se encuentran dentro de Skyteam. Es importante observar que la empresa ha incrementado sus ventas, un factor a tomar en cuenta es la quiebra de su principal competidor en el mercado interno de México, Mexicana de Aviación. Esta quiebra hizo que Aeroméxico operara rutas que eran antes manejadas por Mexicana de Aviación y también permitió que esta fortaleza interna impulsara su expansión internacional.

\section{LATAM}

La unión LAN y TAM trajo importantes beneficios para estas dos empresas, ya que estas eran independientes, cada empresa tenía su propia estrategia y operaciones (Zabala, 2012). El hecho de que estas dos empresas llevaran a cabo esta estrategia trajo como resultado grandes cambios, y además aumento la atracción de clientes debido al aumento de destinos, vuelos y flota aérea (https://www.latam.com).

Lo anterior trajo como resultado que LATAM se convirtiera en una de las aerolíneas más importantes de América Latina. LATAM se unió a diferentes aerolíneas alrededor del mundo para mejorar y coordinar los vuelos internacionales que realizan realizando códigos compartido. Asimismo LATAM desde 2014 forma parte de la alianza OneWorld con otras 13 aerolineas de diferentes partes del mundo, estas son: Aiberlin, American Airlines, British Airways, Cathay Pacific, Finnair, Iberia, Japan Airlines, Malasya, Qantas, Qatar, Roya Jordania, S7 AAirlines y Sri Lanka Airlines. La alianza cuenta una red global de rutas que cubre más de 1,000 destinos en más de 160 países, transferencias más fáciles para pasajeros y acceso de horarios de vuelos de todas las aerolíneas asociadas de "One World".

La empresa ha incrementado sus ventas (USMD) desde el 2010, empezando por $\$ 4,390$ y sucesivamente en el 2011 con un total de $\$ 5,885$, en el 2012 ventas por $\$ 9,710$, en el 2013 ventas por $\$ 12,924.5$, en el año 2014 decremento sus ventas a un total de $\$ 12,093.5$ y sorpresivamente en el año 2015 cerraron con $\$ 9,740$.

Asimismo LATAM cuenta con una importante demanda dentro del mercado interno de Brasil con un gran número de vuelos nacionales a 48 destinos diferentes dentro del país, además más de la mitad de flotas 212 operan en Sudamérica por el corto alcance y potencia cuyo preferencia es tener una mayor flexibilidad de vuelos, permitiéndole servir con efectividad en 
las rutas domésticas y regionales. Se requieren de gran cantidad de flota para la aerolínea, para que desde cada uno de los países en el cual opera pueda sostener la demanda. Actualmente cuenta con un total de 331 aviones tanto como para vuelos nacionales e internacionales de la aerolínea (http://memoria2012.marketinglan.com/flota.html).

\section{Avianca}

Avianca utilizo diferentes estrategias de internacionalización para lograr el crecimiento a lo largo de los años. Entre estas estrategias se encuentra la alianza estratégica con la Sociedad Aeronáutica de Medellín (SAM) y Helicópteros Nacionales de Colombia (HELICOL), las cuales eran compañías muy importantes en el este sector de la aviación colombiana. Con esta alianza estratégica buscaron fortalecer principalmente a Avianca, en la cual invirtieron todo el capital en esta aerolínea para lograr crecer más (http://www.aviancacargo.com). Avianca logró una nueva alianza estratégica con TACA, con la cual buscaban posicionarse en el mercado, tener mayor capacidad para crecer y así lograr tener la mayoría del mercado.

Avianca pertenece a la alianza entre aerolíneas llamada "Star Alliance", formada por 28 aerolíneas (http://www.staralliance.com), con esta alianza la empresa logro consolidar su estrategia para enfrentar a la competencia. Por ejemplo, con la alianza se obtuvieron diversos beneficios para los pasajeros como para la aerolínea, entre ellos el ofrecer una mayor frecuencia de vuelos y destinos, ya que uno de los beneficios de pertenecer a esta alianza es compartir vuelos y espacios en los aviones para poder tener más destinos disponibles (http://www.avianca.com/mx/es/nuestracompania/algunos-aliados/star-alliance).

\section{Discusión de resultados}

Los principales resultados de este trabajo arrojan que las tres aerolíneas siguen patrones de internacionalización similares. Además es importante tomar en cuenta que la calidad en los servicios es más relevante que en los bienes tangibles. Por lo tanto el control de los procesos es un asunto clave para la satisfacción de los usuarios de los servicios. En el caso particular de Aeroméxico centra mucho de sus capacidades en el mercado interno en 
México, dado que es la única aerolínea mexicana que cubre la totalidad del país. Además la empresa no tiene un competidor de sus mismas dimensiones en el mercado interno de México. Estas dos situaciones confirman que la empresa compite internacionalmente en algunas rutas dado que es muy sólida en el mercado interior de su país. Además Aeroméxico tiene importante presencia en el mercado de Estados Unidos cubriendo 15 destinos y 272 vuelos semanales.

En lo referente a Avianca tiene un patrón similar de internacionalización, sin embargo, se diferencia de Aeroméxico en que Colombia es un país territorialmente con menor extensión territorial y con menos aeropuertos que en México. Avianca es una empresa importante en centro y Sudamérica ya que cubre 24 destinos dentro de la región con un total de 293 frecuencias semanales, siendo los países de la región cercana sus principales destinos.

LATAM contrario a Aeroméxico y Avianca es una empresa que surgió recientemente de una fusión de dos aerolíneas. Esto la llevo a concentrar las ventajas competitivas de ambas, convirtiéndola en una de las más importantes de la región. Además por la ubicación de Chile esta empresa es la única que ofrece rutas a los 5 continentes con rutas a Sudáfrica y Australia, además a las tradicionales de Europa y Asía. Asimismo sus destinos se concentran 10 ciudades de América Latina, estos incluyen 98 frecuencias semanales. Además LATAM es la única que ofrece un gran número de vuelos internos en un país diferente al suyo como lo hace en Brasil.

Otra de las principales similitudes de las tres aerolíneas es que sus principales destinos se encuentran en el continente Americano. Además de tener presencia en Europa y Asia. Avianca y Aeroméxico ofrecen una gran variedad de destinos a Centroamérica. Además Aeroméxico es la aerolínea que tiene mayor cantidad de destinos al continente Asiático, esto dado por la ubicación geográfica de México.

\section{Conclusiones}

El objetivo principal de este trabajo fue analizar las estrategias de internacionalización de las aerolíneas latinoamericanas Aeroméxico, Avianca y LATAM. Además la principal contribución de este trabajo es aumentar la literatura sobre multinacionales de América Latina en el sector de servicios 
utilizando evidencia empírica y un abordaje del problema de investigación distinto a lo que se ha hecho antes. Asimismo se identificó que las aerolíneas no se ajustan al modelo gradual y secuencial de Uppsala debido a tres factores: la naturaleza del sector aéreo, la intangibilidad de los servicios que brindan, el tamaño de su mercado interno reducido (como lo es Colombia y Chile) y su ubicación geográfica cercana a mercados mucho más grandes (Aeroméxico con Estados Unidos y LATAM con Brasil y Argentina). Los factores anteriores inciden en que las tres aerolíneas estudiadas presentan procesos de internacionalización acelerados. En el caso de la teoría del paradigma ecléctico las empresas estudiadas se ajustan a esta teoría.

En este orden de ideas y de acuerdo con la información recopilada la conclusión general de este trabajo es que las tres aerolíneas utilizan estrategias de internacionalización similares, sin embargo presentan algunas diferencias dada sus condiciones como empresa y el entorno de su país de origen. La principal estrategia de internacionalización utilizada por las aerolíneas son las alianzas estratégicas. Esto puede observarse dado que las tres empresas forman parte de alianzas estratégicas internacionales (One World, SkyTeam y Star Alliance) con otras aerolíneas donde comparten recursos para poder brindar mejores servicios a sus pasajeros.

Estas alianzas requieren además cierto grado de presencia local. Esto genera que las tres aerolíneas tengan que realizar inversiones directas en los países donde tienen operaciones para poder cumplir con los estándares de calidad y servicios que les permita competir en el mercado.

Asimismo de acuerdo con los datos obtenidos las tres empresas son fuertes en sus mercados locales, esto hace que gracias a esta fortaleza puedan desarrollar ventajas que les permiten competir en mercados internacionales. La que más se beneficia de esto es Aeroméxico ya que el mercado interno de México es el más grande los tres países. En el caso de Avianca y LATAM sus mercados locales son más pequeños, pero han podido captar mercados internos, gracias a la operación de vuelos internos en países vecinos como Ecuador y Perú (Avianca) y Argentina y Brasil (LATAM).

En lo referente a la relación de las aerolíneas con las teorías de la internacionalización, se ejemplifica que no siguen un proceso gradual y secuencial como lo señala Johanson y Valhne (1977). Probablemente sea dado la propia naturaleza de los servicios que proporciona, estas empresas presentan más similitudes con las empresas "born global", las cuáles son 
aquellas que desde su fundación buscan obtener beneficios de operar en múltiples países (Sultan \& Wong, 2011). Asimismo tienen una mayor relación con lo propuesto por Dunning (1977) con la Teoría OLI, sobre todo en la L (Localización) donde estas empresas aprovechan sus ventajas locales para poder internacionalizarse con éxito ya que todas ellas son las más importantes en sus respectivos países de origen. Además las aerolíneas estudiadas cumplen la premisa clásica de internacionalización ya que se expanden internacionalmente a lugares cercanos geográficamente y con afinidad cultural.

Como futuras líneas de investigación es importante analizar, dado que es un sector de alta tecnología, ¿cómo estas empresas internacionalizan sus capacidades tecnológicas en los países donde operan?. Además determinar cuáles son las causas por las cuáles concentra sus operaciones en países cercanos geográficamente y analizar como la fortaleza en su mercado local ha incidido en su internacionalización. Asimismo es importante analizar a fondo el papel que ha jugado en su internacionalización las diversas alianzas estratégicas con otras aerolíneas en su desempeño financiero. Finalmente, al ser el sector aéreo uno de los más regulados del mundo, es importante analizar el papel de las políticas y regulaciones nacionales e internacionales que inciden en las operaciones de las aerolíneas.

\section{Referencias}

Alserus, M. \& Tykesson, D. (2011). The uppsala model's applicability on internationalization processes of European SMEs, today. Lund, Sweden: Lunds Universitet.

Banco Interamericano de Desarrollo. (2016). Temas actuales para América Latina y el Caribe, Transporte Aéreo, Regulación y Economía. Recuperado de: https://publications.iadb.org/bitstream/handle/11319/7607/Transporte-AereoTemas-Actuales-para-America-Latina-y-El-Caribe-Regulacion-yEconomia.pdf?sequence $=13$.

Capar, N. \& Kotabe, M. (2003). The relationship between international diversification and performance in service firms, Journal of International Business Studies, 34(4), 345355

CEPAL (2017). Transporte aéreo como motor del desarrollo sostenible en América Latina y el Caribe: Retos y propuesta de política, Boletín FAL, 359, 1-11.

Cruz-García, A. \& Puerta, S. (2012). Internacionalización del sector aéreo colombiano. (Tesis de grado). Universidad del Rosario, Bogotá, Colombia. 
Danciu, V. (2012). Models of internationalization of the business: A diversity- based approach, Management \& Marketing, 7(2), 29- 42.

Delpiano, R. (2009). 80 años de LAN. Recuperado de: http://www.desdescl.com/2009/03/80anos-lan-airlines.html

Driffield, N., Pereira, V. \& Temouri, Y. (2017). Does offshore outsourcing impact home employment? Evidence from service multinationals, Journal of Business Research, Early online.

Dunning, J. H. (1988). The eclectic paradigm of international production: A restatement and some posibles extensions, Journal of International Business Studies, 19(1), 1- 31.

Eden, L. \& Dai, L.(2010). Rethinking the O in Dunning's OLI/ Eclectic Paradigm. Multinational Business Review, 18(1), 13-34.

Faulconbridge, J. \& Muzio, D. (2016). Global profesional service firms and the challenge of institutional complexity: "Field relocation" as a response strategy, Journal of Management Studies, 53(1), 89-124.

Franco- Arroyave, C., Martins- Cheze, A., Siegel, A. and Diaz- Vazquez, J.C. (2014). Internationalization strategies of a multilatina in the service sector: Avianca- Taca holding S.A. case study, Ad-Minister, 25, 79-96.

Gleich, W.; Schmeisser, B. \& Zschoche, M. (2016). The influence of competitios on international sourcing strategies in the service sector, International Business Review, 26(2), 279-287.

Gustafsson, T. \& Zasada, D. (2011). Explanatory power of internationalization theories, a case study of Norweigan SMEs targeting developing countries. Recuperado de: https://www.duo.uio.no/bitstream/handle/10852/12885/GustafssonZasada. pdf?sequence $=2 \&$ isAllowed $=y$

Johanson, J. \& Vahlne, J.E. (1977). The internationalization process of the firm: A model of knowledge development and increasing foreign markets commintments, Journal of International Business Studies, 8(1), 23- 32.

LATAM (2016). Grupo LATAM Airlines fortalece su conexión con Estados Unidos e inicia operaciones de vuelo directo a Los Ángeles todo el año (Comunicados de prensa, octubre 19). Recuperado de http://www.latam.com

LATAM (2015). Memoria anual LATAM 2015. Santiago de Chile: LATAM Airlines.

LAN (2013). Información sobre LAN Airlines. Santiago de Chile: LAN Airlines.

LATAM (2014). LATAM Airlines Group revisa sus perspectivas para 2014, Relación con Inversionistas LATAM (Noticias, julio 08). Recuperado de http://www.latamairlinesgroup.net

$\mathrm{Li}$, J. \& Guisinger, S. (1992). The globalization of service multinationals in the "Triad" regions: Japan, Western Europe and North America, Journal of International Business Studies, 23(4), 675- 695 .

López-Morales, J. S. (2018). Multilatinas: A systematic literatura review, Review of International Business and Strategy, 28(3/4), 331-357. 
López-Morales, J.S. \& Nava- Aguirre, K. M. (2018). The road to internationalization of a Latin state company: The case of PEMEX, Journal Globalization, Competitiveness \& Governability, 12(2), 97-114.

Ministerio de Comercio (2011). Importancia de los servicios. Bogotá: Ministerio de Comercio, Industria y Turismo

Rammal H. \& Rose E. (2014). New perspectives on the internationalization of service firms, International Marketing Review, 31(6), 550-556.

Ruiz, M. (2012). Lan y Tam se fusionan en Latam, la mayor aerolínea de Latinoamérica, Recuperado 9 de Octubre del 2017, de https://elpais.com/economia/ 2012/06/22/actualidad/1340389912_308875.html

Rugman, A. M. (2010). Reconciling internationalization theory and the Eclectic Paradigm, Multinational Business Review, 18 (2), 1- 12.

Rivas, R.M. \& Mayorga, D. (2011). Internationalization of Peruvian cuisine: An analysis of internationalization strategies of Peruvian restaurants, INNOVAR. Revista de Ciencias Administrativas y Sociales, 21 (39), 205- 216.

Sultan, P. \& Wong, H.W. (2011). The success of born global firms: a conceptual model, Journal of Global Business Advancement, 4, 3, 224-241.

OMC. (2005). Comercio de servicios de transporte aéreo: Evolución reciente y asuntos de política, En OMC (Ed.) Informe sobre el comercio mundial. Ginebra: Organización Mundial del Comercio, 237-292.

Reddy, K.S. \& Agarwal, K. (2012). Designing case studies from secondary sources. A conceptual framework, International Management Review, 8 (2), 63-70.

Wang, L. \& Chen, H. (2015). A review and prospect on internationalization process of service multinationals corporations, Journal of Service and Management, 8, 77- 84

World Trade Organization. (2013). The general agreement on trade in services. An introduction. WTO. Retrieved from: http://www.wto.org/english/tratop_e/ serv_e/gsintr_e.pdf

Garcia, X. (2017). Aeroméxico mantiene más destinos internacionales que nacionales. Noticias de la Industria Turistica, 14 de octubre del 2017. Recuperado de: https:/www.nitu.mx/index.php/2017/03/29/aeromexico-mantiene-mas-destinosinternacionales-que-nacionales/

Yin, Robert (2009). Case Study Research: Design and Methods ( $4^{\mathrm{a}}$ ed.). California: Sage.

Zabala, J. L (2012). LATAM Airlines Group: la fusión de dos gigantes, Recuperado 21 de Agosto del 2017, de http://www.academia.edu/8336190/LATAM_Airlines_ Group_La_fusio_n_de_dos_gigantes. 